\section{La Révolution française}

Cahiers de l'Institut d'histoire de la Révolution française

$9 \mid 2015$

Citoyenneté, république, démocratie dans la France de la Révolution

\title{
Monsieur André Aubusson, le secrétaire de grand mérite du Comité de salut public ou gérer la correspondance sous la première année de la République (deuxième partie)
}

Monsieur André Aubusson, Secretary of great merit of the Committee of Public

Safety, or the control of correspondence in the first year of the Republic (part 2)

Maria Betlem Castellà i Pujols

\section{(2) OpenEdition}

\section{Édition électronique}

URL : http://journals.openedition.org/lrf/1447

DOI : $10.4000 /$ Irf. 1447

ISSN : 2105-2557

Éditeur

IHMC - Institut d'histoire moderne et contemporaine (UMR 8066)

Référence électronique

Maria Betlem Castellà i Pujols, « Monsieur André Aubusson, le secrétaire de grand mérite du Comité de salut public ou gérer la correspondance sous la première année de la République (deuxième partie) », La Révolution française [En ligne], 9 | 2015, mis en ligne le 16 novembre 2015, consulté le 19 avril 2019. URL : http://journals.openedition.org/Irf/1447 ; DOI : 10.4000/Irf.1447

Ce document a été généré automatiquement le 19 avril 2019

(c) La Révolution française 


\title{
Monsieur André Aubusson, le secrétaire de grand mérite du Comité de salut public ou gérer la correspondance sous la première année de la République
}

Monsieur André Aubusson, Secretary of great merit of the Committee of Public Safety, or the control of correspondence in the first year of the Republic

\author{
Maria Betlem Castellà i Pujols
}

\section{NOTE DE L'AUTEUR}

Étant donné l'extension originale de ce travail, il a été divisé en deux parties : une première partie concernant l'Assemblée nationale constituante et l'Assemblée législative, et une deuxième partie concernant la Convention nationale. La première partie a été publiée sous le titre Maria Betlem CASTELLÀ I PUJOLS, « Monsieur André Aubusson, l'homme qui sait tout, ou gérer la correspondance sous les assemblées parlementaires », $L a$ Révolution française [En ligne], 8 | 2015, mis en ligne le 24 juin 2015, Consulté le 11 octobre 2015. URL : http://lrf.revues.org/1312. La deuxième partie concerne le présent travail. Par ailleurs, cette étude a été réalisée dans le cadre du Grup d'Estudi de les Institucions i de les Cultures Polítiques (segles XVI i XXI) (2014 SGR 1369) et grâce à un contrat Beatriu de Pinós modalité B de l'Agence de Gestion des Aides universitaires et de Recherche (AGAUR) de la Generalitat de Catalogne (2010-BP-00124). 


\section{Introduction}

1 Si l'on prend en compte les liens existant entre pouvoir et information, on s'étonne du peu d'attention accordée par les historiens à la manière dont les députés ont géré la correspondance reçue par les différentes assemblées parlementaires entre 1789 et 1795, ainsi qu'aux individus, comités et commissions qui ont joué un rôle de filtre sur les informations reçues.

2 Ce manque d'attention pour une procédure qui intervient directement sur les résultats du débat parlementaire n'a pas permis d'observer tous les changements que la Convention nationale a apportés quant à la façon avec laquelle l'Assemblée nationale constituante et l'Assemblée législative avaient géré les milliers de lettres que les citoyens, les administrations, les tribunaux, les militaires, les sociétés ou les comités révolutionnaires leur avaient envoyées ; pas davantage la perte des compétences subie par le président de l'Assemblée à cause d'un nouveau système d'information mis en place par les conventionnels au début du mois d'octobre, ni l'impact que l'établissement du Comité de salut public eut sur ce nouveau système, en le transformant de fond en comble.

3 En fait, on demeure curieux d'observer comment, parmi les nombreux travaux consacrés au Comité de salut public, depuis sa dissolution jusqu'aujourd'hui ${ }^{1}$, mesurant son pouvoir, analysant ses compétences et ses travaux ainsi que les luttes politiques en son sein et autour de lui, aucune analyse n'a été faite à propos de l'incidence que ce comité eut sur les filtres établis par la Convention nationale sur la correspondance reçue, ainsi que sur les «porters » - concept décrit par Kurt Lewin pour désigner ceux qui occupaient des postes-clés pour faire circuler les informations.

4 Le premier objectif du présent article, qui est en fait la deuxième partie d'un autre travail publié sous le titre: "Monsieur André Aubusson, l'homme qui sait tout, ou gérer la correspondance sous les assemblées parlementaires ", n'est autre que de montrer, en suivant la trajectoire professionnelle de M. André Aubusson, ancien militaire devenu secrétaire-commis en $1789: 1$ ) comment la correspondance est gérée sous la Convention nationale, et 2) qui s'occupe de cette gestion. Le deuxième objectif, qui n'est pas moins important que le premier, est de mettre au premier plan le parcours professionnel de M. André Aubusson qui, ayant été chef du Bureau de correspondance et dépêches et adjoint au Secrétariat de la Présidence de l'Assemblée nationale constituante, de la législative et de la Convention depuis le $1^{\text {er }}$ juillet 1789 , devient secrétaire principal du Comité de salut public sous la Convention nationale le 13 septembre 1793 et chef de la division du Secrétariat général du Directoire exécutif le 13 brumaire de l'an IV ${ }^{2}$. Le troisième et dernier objectif n'est autre que de montrer la continuité que l'on peut apprécier d'un même personnel administratif dans le cadre des bureaux et des comités qui s'occupent de gérer la correspondance reçue sous les différentes assemblées parlementaires. Les députés sont renouvelés de temps en temps, mais les secrétairescommis qui y travaillent, certains d'entre eux depuis les premiers jours de l'Assemblée nationale constituante, ne le sont pas, configurant ainsi un corps d'employés expérimentés qui conservent leurs fonctions au long des trois premières assemblées, et parfois, dans les années qui suivent. 


\section{Deuxième Partie : (Septembre 1792 - 18 Brumaire an VIII)}

\section{Comment gérer la correspondance : entre le droit sacré de pétition et la défense de la démocratie représentative}

5 Si les premiers jours de l'Assemblée législative furent marqués par les interventions d'Yves Marie Audrein, François Chabot et Georges Auguste Couthon dénonçant les filtres établis sur la correspondance reçue à l'époque de l'Assemblée nationale constituante, et par la volonté des nouveaux élus de se faire lire un extrait de toute la correspondance reçue pour conserver le droit sacré de pétition et ménager en outre le temps de l'Assemblée, les premiers jours de la Convention nationale ne le furent pas par ces aspirations. Aucune intervention ne fut faite pour se plaindre des filtres établis sur la correspondance dans le cadre de l'Assemblée législative à partir du 3 novembre 1791 ou pour se faire lire toutes les pétitions et les adresses que les commettants pouvaient adresser à l'Assemblée. En fait, au début de l'automne 1792, les députés semblaient être d'accord sur le fait qu'ils ne disposaient pas d'un temps suffisant pour lire tout ce que les commettants voulaient leur communiquer. Plus encore, ils semblaient être d'accord sur le fait que les citoyens devaient s'adresser directement à leurs autorités administratives et judiciaires pour présenter leurs pétitions particulières, étant donné que ceux qui y travaillaient n'étaient pas hostiles aux intérêts du peuple sinon bien au contraire leurs défenseurs :

Aujourd'hui que la liberté et l'égalité triomphent de l'attaque qui leur a été constamment livrée jusque dans leurs premiers éléments; que les corps administratifs et judiciaires vont être mieux épurés encore par des renouvellements indispensables autorisés par la loi, il est bien désirable que les citoyens connaissent enfin la hiérarchie des pouvoirs, si nécessaire au repos et à l'avantage de tous. On ne saurait mieux prouver son patriotisme, qu'en ne dérobant pas, sans une grande et urgente nécessité aux fondateurs des lois, un seul de leurs instants ${ }^{3}$.

6 Pour Constant Joseph Eugène Gossuin, membre du Comité des pétitions de l'Assemblée législative, et président du nouveau Comité des pétitions et de correspondance sous la Convention nationale, il fallait respecter le droit sacré de pétition, mais ne pas permettre que ceux qui s'adressaient au corps législatif utilisent ce droit pour faire entendre leurs avis ou les discours que l'amour-propre ou l'agitation, selon les mots de Gossuin luimême, leur auraient dictés :

Sans contredit, le droit de pétition est sacré ; s'environner des citoyens, entendre leurs justes plaintes, y répondre avec aménité et sans retard, c'est un bonheur qui était autant méconnu des rois et de leurs agents, qu'il est ambitionné par chacun de vous; mais il ne faut pas que ce droit si utile dégénère en abus. Soyons avares de notre temps ; montrons-nous fermes envers ceux qui, après de longs discours dictés souvent par l'amour-propre ou par l'agitation, et prononcés avec emphase à la barre, ne nous ont rien dit; prêtons au contraire beaucoup d'attention aux citoyens victimes de quelques malversations, enfin aux citoyens paisibles; et on pourrait citer pour exemple ceux du faubourg Saint-Antoine, dont le respect pour la loi a toujours égalé la franchise ${ }^{4}$.

7 Au début de la République naissante, et selon un rapport qui fut présenté par Gossuin à la Convention nationale le 23 octobre 1792, il semblait clair que les députés étaient disposés 
à écouter les justes plaintes de leurs concitoyens. Cependant, en ce qui concernait les longs discours que ceux-ci pouvaient faire à l'occasion, cela n'était pas si clair. Comme ne l'était pas non plus le fait qu'ils dussent écouter tous les vœux des commettants adressés à l'Assemblée pour présumer de la volonté générale et légiférer. Pour Gossuin la lecture des pétitions, des adresses, des dons patriotiques et des lettres particulières ralentissait les travaux de l'Assemblée :

Vos séances sont journellement de six heures ; si ce temps était employé selon votre vœu, bientôt on bénirait vos travaux; mais une longue lecture de pétitions, d'adresses, de dons patriotiques, de lettres particulières, souvent des motions incidentes qui se croisent et se prolongent sur l'un ou l'autre de ces objets agitent l'Assemblée; les heures s'écoulent et le travail va lentement ${ }^{5}$.

8 À la fin du mois d'octobre, les conventionnels ne reprenaient pas les interventions faites par les députés de l'Assemblée législative pour se faire lire toute la correspondance reçue; ils reprenaient plutôt celles que Pierre Bernard d'Héry ou que Jean François Blanchon avaient faites pour interdire aux commettants de discuter dans l'enceinte parlementaire sur les grands intérêts de la nation pour lesquels eux seuls avaient été appelés :

Je commence par reconnaître - disait Blanchon de la Charente le 23 octobre 1791 que le droit de pétition appartient à tous les individus français, mais je sais bien aussi que le droit de pétition n'est pas de délibérer avec les membres de l'Assemblée nationale sur les objets dont elle s'occupe. Le droit de pétition consiste à demander ce qui est nécessaire à un individu ou à plusieurs individus, mais non pas à avoir l'initiative sur chaque membre de l'Assemblée nationale; ce sont eux seuls qui doivent discuter et délibérer dans cette Assemblée pour préparer la loi qui doit s'y rendre ${ }^{6}$.

9 C'est dans ce contexte, où la démocratie représentative prévaut sur la démocratie directe ${ }^{7}$ , qu'il faut inscrire le projet présenté par Gossuin le 23 octobre 1792 ; projet selon lequel seules les lettres des commissaires envoyés en mission, des ministres et des généraux seraient lues à l'Assemblée :

Art. $1^{\text {er }}$ : les lettres des commissaires aux armées, des ministres et des généraux seront lues immédiatement après le procès-verbal ; il sera également donné lecture sans retard de celles qui arriveront dans le cours de la séance.

Art. 2: toutes ces lettres seront aussitôt renvoyées aux comités compétents. La discussion de leur contenu ne pourra s'ouvrir dans la séance qu'en vertu d'un décret ${ }^{8}$.

10 Aucune lettre particulière ne serait lue aux députés ; pas davantage que les pétitions, les adresses ou les mémoires :

Art. 3 : Aucune lettre particulière ne sera lue à la Convention.

Art. 4 : Les adresses, pétitions et les lettres remises officiellement à la Convention, autres que celles mentionnées en l'article $1^{\mathrm{er}}$, seront, chaque jour, déposées par le soin du bureau, au comité des pétitions et correspondance, qui en tiendra registre, en fera l'analyse, en rendra compte tous les jeudis et dimanches, et proposera les renvois 9 .

11 Les dons ne seraient présentés par le Comité des pétitions et de correspondance que les dimanches:

Art. 5 : Le comité des pétitions et de correspondance dressera chaque semaine un bordereau des dons patriotiques; il en sera fait lecture le dimanche; toute autre forme de proclamation sur cet objet est rigoureusement interdite ${ }^{10}$.

12 Aucun pétitionnaire ne lirait sa pétition sans l'avoir communiquée au préalable au Comité des pétitions et de correspondance : 
Art. 6 : Tout pétitionnaire qui désirera paraître à la barre sera tenu de se présenter au comité, d'y énoncer l'objet de sa pétition et de s'y faire inscrire ${ }^{11}$.

Et, en dernier ressort, les pétitionnaires venus de l'extérieur de la capitale seraient les premiers à présenter leurs pétitions :

Art. 7 : L'ordre d'admission des pétitionnaires à la barre suivra invariablement, chaque dimanche, celui de leur inscription au registre; il n'y aura exception que pour ceux résidant hors des barrières de Paris qui, en raison de leur éloignement, seront admis les premiers. Le tableau en sera toujours remis à temps à la commission centrale, pour régler son travail ${ }^{12}$.

14 Le 11 novembre 1792, sans la moindre discussion, les députés adoptèrent le projet de décret présenté par Gossuin.

\section{II. Ôter les compétences du président de l'Assemblée : un dommage collatéral du nouveau système d'information?}

Gossuin n'expliqua jamais ouvertement aux députés les dommages collatéraux que les "porters» établis sous l'Assemblée nationale constituante et l'Assemblée législative allaient subir avec l'adoption de son projet de décret. D'abord, le Bureau des renvois et de correspondance cesserait de gérer la correspondance reçue; ensuite, M. Aubusson perdrait la compétence de décider de la destination pour chacune des lettres qui passaient par ses mains, c'est-à-dire de décider des lettres qui pouvaient être susceptibles d'être lues entièrement aux députés et de celles qui devaient être résumées; des lettres qui devaient être renvoyées aux différents comités et de celles qui devaient être communiquées immédiatement, du fait de l'importance de leur contenu ou de leur urgence, au président ou aux comités de l'Assemblée. Il n'expliqua jamais ouvertement aux députés qu'avec l'adoption de l'article 4 de son projet de décret toute la correspondance reçue par la Convention nationale passerait par le comité qu'il présidait.

Or, ni le Bureau des renvois et de correspondance ni M. Aubusson ne furent les seuls à perdre des compétences en faveur du Comité des pétitions et de correspondance. Le président de l'Assemblée perdit la compétence de communiquer ses ordres quant à la destination des lettres reçues. Plus encore, il perdit la compétence d'avoir sous sa direction le Bureau des renvois et de correspondance, qu'il avait sous son contrôle depuis le début du processus révolutionnaire, et de décider des pétitionnaires qui pouvaient lire leurs pétitions à l'Assemblée. À partir du 11 novembre 1792, il demeurait avec la seule compétence de gérer le débat parlementaire. Il ne pourrait plus décider ni de ce qui serait lu à l'Assemblée ni de qui interviendrait pour communiquer ses points de vue ou ses pétitions aux députés du corps législatif.

17 Les compétences que Gossuin attribuait au comité qu'il présidait - et dont il faisait partie depuis son établissement sous l'Assemblée législative - étaient considérables. Grâce à l'adoption de son projet de décret, le Comité des pétitions et de correspondance devenait le premier filtre sur la correspondance reçue, et donc le premier filtre sur la voix des commettants. Seules les lettres des députés envoyés en mission, des ministres et des généraux seraient lues. Le reste de la correspondance serait renvoyé directement aux comités par le Comité des pétitions et de correspondance ou ferait partie d'un résumé que ce comité présenterait à la Convention. 


\section{Le Comité des pétitions et de correspondance : le centre de toutes les affaires de la Convention nationale}

18 Avec l'adoption du projet de décret de Gossuin, le Bureau des renvois et de correspondance et M. Aubusson perdirent leurs compétences le 11 novembre 1792, trois ans et trois mois après les avoir obtenues. Toutefois, cinq jours après, le Comité des pétitions et de correspondance décida d'incorporer dans ses bureaux celui des renvois et de correspondance ${ }^{13}$. Avec cette décision, le Comité des pétitions et de correspondance cessait d'avoir deux sections pour en avoir trois: la Section des pétitions, qui avait la compétence de gérer les pétitions, les adresses et les mémoires envoyés par les commettants; la Section de correspondance, qui avait la compétence de rédiger le bulletin national et de correspondre avec les députés envoyés en mission; et la Section des renvois et dons patriotiques, dans laquelle il y aurait le Bureau des renvois et de correspondance et M. Aubusson, pour s'occuper des articles 4 et 5 du projet de décret présenté par Gossuin.

Pendant une semaine, du 16 au 23 novembre 1792, les députés qui constituaient le Comité des pétitions et de correspondance envisagèrent divers projets afin d'organiser les différentes sections du comité. Le 16 novembre, ils décidèrent qu'il y aurait trois sections : la Section des pétitions, la Section des renvois et dons patriotiques et la Section de correspondance ${ }^{14}$. La première section aurait cinq secrétaires-commis et six commissaires inspecteurs (députés du Comité); la deuxième section aurait six secrétaires-commis et six commissaires inspecteurs; et la troisième section aurait huit secrétaires-commis et six commissaires inspecteurs. Le comité aurait également un président et deux secrétaires ${ }^{15}$.

Cependant, le 21 novembre, les députés du Comité des pétitions et de correspondance changèrent d'opinion quant au nombre total de sections, et ils décidèrent qu'il y en aurait deux : la Section des pétitions et la Section de correspondance. Ce jour-là, ils décidèrent par ailleurs des secrétaires-commis qui occuperaient chacune des sections ${ }^{16}$. MM. Garnier, Hussenet, Chaulay, Dupuis, Chachoin, Jennesse, Atrux, Vaillant ${ }^{17}$ et Henry occuperaient la Section des pétitions, alors que MM. Fourcade, Becquier, Jourdan, Douet, Larivelle, Rimoneaux, Doumengeat et Renvoizé occuperaient celle de correspondance. En ce qui concernait M. Ducroisy, il s'occuperait des dons patriotiques alors que MM. Aubusson et Thibault s'occuperaient de faire le tri de toute la correspondance reçue ${ }^{18}$.

21 Un mois après le début de l'activité de la Convention nationale, le Comité des pétitions et de correspondance continuait ainsi à confier à $\mathrm{M}$. Aubusson le tri de la correspondance qu'il recevait. Les députés qui légiféraient pouvaient changer mais l'homme qui lisait toutes les lettres pour la première fois et décidait de leur destination continuait à être le même trois ans et quatre mois après le début du processus révolutionnaire. Le mérite et l'expérience étaient des valeurs reconnues ${ }^{19}$.

Enfin, le 23 novembre 1792, les députés du Comité des pétitions et de correspondance décidèrent qu'il y aurait trois sections: la Section des pétitions à laquelle tous les pétitionnaires s'adressaient afin d'être admis à la barre ou pour faire parvenir leurs pétitions à qui devait les gérer :

C'est à la section des pétitions que tous les pétitionnaires indistinctement se rendent pour leur admission à la barre ou le renvoi de leurs demandes aux différents comités compétents, aux ministres [et aux] corps administratifs. Leurs 
adresses, lettres, missives et autres pièces quelconques remises sur le bureau de la Convention nationale y sont chaque jour enregistrées ${ }^{20}$.

Convention nationale mais il cessa de maintenir son indépendance et son intégrité dans le cadre du Comité des pétitions et de correspondance. Il fut cantonné au tri de la correspondance reçue et vit dispersés, entre les différentes sections du Comité des pétitions et de correspondance, cinq des six secrétaires-commis dont il disposait depuis ses débuts.

Si on lit le procès-verbal de ce comité, on observe comment, le 16 novembre 1792 , le Comité des pétitions et de correspondance fut structuré en trois sections correspondant aux trois organismes qui le composaient. Le Comité des pétitions de l'Assemblée législative s'était converti en une section, où travaillaient ses cinq secrétaires-commis : MM. Garnier, Hussenet, Chaulay, Dupuis et Chachoin; le Bureau des renvois et de correspondance de l'Assemblée législative s'était transformé en Section des renvois et dons patriotiques, et il conservait ses six secrétaires-commis : MM. Aubusson, Renvoizé, Atrux, G. Vaillant, Le Harivel et Henri; et la Commission de correspondance de l'Assemblée législative était devenue section avec huit secrétaires-commis qui, par élimination, devaient être: MM. Jennesse, Thibault, Doumengeat, Fourcade, Becquier, Jourdan, Douet et Rimoneaux ${ }^{24}$. 
ependant, cette structure qui maintenait trois organismes séparés, le Comité des pétitions, la Commission de correspondance et le Bureau des renvois et de correspondance, ne dura pas longtemps. Le 21 novembre 1792, le Comité des pétitions et de correspondance décida, en effet, de distribuer cinq des six secrétaires-commis du Bureau des renvois et de correspondance entre la Section des pétitions et la Section de correspondance. MM. Atrux, G. Vaillant et Henri qui, depuis le début de l'activité de l'Assemblée nationale constituante, se consacraient à l'analyse de la correspondance passèrent à la Section des pétitions, alors que MM. Le Harivel et Renvoizé, qui étaient chargés des registres depuis l'été 1789 , passèrent à la Section de correspondance ${ }^{25}$. Le seul qui demeura dans l'ancien Bureau des renvois et de correspondance, reconverti en Section des renvois et dons patriotiques, fut $\mathrm{M}$. Aubusson. Il y demeura, aux côtés de deux autres secrétaire-commis, l'un dédié à la gestion des dons patriotiques, M. Ducroisi, et l'autre dédié à l'aider, M. Thibault, afin d'effectuer le même travail qu'il faisait depuis le mois de juillet 1789 :

Ils seront chargés de trier les lettres, pétitions et mémoires adressés par la poste à la Convention, de remettre au Président les objets dont il sera nécessaire de faire donner la lecture à l'Assemblée et de recueillir le reste pour être envoyé chaque jour à la section des pétitions ${ }^{26}$.

Cette réorganisation du personnel de base du Comité des pétitions et de correspondance qui peut sembler sans importance, supposait en fait une quasi défaite de l'ancien Bureau des renvois et de correspondance. Des neuf secrétaires-commis qu'il avait eus sous l'Assemblée nationale constituante seul M. Aubusson continuait à y travailler, deux mois après le début de la Convention nationale.

\section{Des professionnels expérimentés : les secrétaires-commis du Comité des pétitions et de correspondance}

M. Aubusson ne fut pas le seul secrétaire-commis à être maintenu dans ses fonctions depuis le mois de juillet 1789 . Si l'on tient compte des secrétaires-commis qui travaillaient dans le Comité des pétitions et de correspondance à l'automne 1792, on peut observer que cinq des six secrétaires-commis qui avaient travaillé dans le Comité des rapports de l'Assemblée nationale constituante, gérant toute l'information relative à l'administration et à la police ainsi que tous les mémoires, les demandes, les adresses et les pétitions des commettants, continuaient à effectuer la même tâche trois ans et trois mois après le début du processus révolutionnaire ${ }^{27}$. Ils étaient passés du Comité des rapports au Comité des pétitions, et du Comité des pétitions à la Section des pétitions du Comité des pétitions et de correspondance ${ }^{28}$. Il se produisit la même chose avec le personnel du Bureau des renvois et de correspondance. Des neuf secrétaires-commis qui avaient travaillé au Bureau de correspondance et au Bureau des renvois de pièces sous l'Assemblée nationale constituante, six continuaient à travailler au Bureau des renvois et de correspondance à la mi-novembre 1792. (Voir le tableau 1)

Tableau 1

Secrétaires-commis du Bureau des renvois et de correspondance

\begin{tabular}{|l|l|l|l|l|}
\hline 28-08-91 & $22-04-92$ & $15-08-92$ & $05-11-92$ & $21-11-92$ \\
\hline
\end{tabular}

La Révolution française, 9 | 2015 


\begin{tabular}{|c|c|c|c|c|}
\hline $\begin{array}{l}\text { Aubusson } \\
\text { (B. } \\
\text { correspondance) }\end{array}$ & $\begin{array}{l}\text { Aubusson } \\
\text { (B. correspondance, } \\
\text { renvois et scrutins) }\end{array}$ & $\begin{array}{l}\text { Aubusson } \\
\text { (Secrétariat de } \\
\text { l'Assemblée) }\end{array}$ & $\begin{array}{l}\text { Aubusson } \\
\text { (Don } \\
\text { patriotique) }\end{array}$ & $\begin{array}{l}\text { Aubusson } \\
\text { (Section renvois et } \\
\text { dons patriotiques) }\end{array}$ \\
\hline $\begin{array}{l}\text { Férès } \\
\text { (B. } \\
\text { correspondance) }\end{array}$ & $\begin{array}{l}\text { Férès } \\
\text { (B. correspondance, } \\
\text { renvois et scrutins) }\end{array}$ & & & \\
\hline $\begin{array}{l}\text { Renvoizé } \\
\text { (B. } \\
\text { correspondance) }\end{array}$ & $\begin{array}{l}\text { Renvoizé } \\
\text { (B. correspondance, } \\
\text { renvois et scrutins) }\end{array}$ & $\begin{array}{l}\text { Renvoizé } \\
\text { (Secrétariat de } \\
\text { l'Assemblée) }\end{array}$ & $\begin{array}{l}\text { Renvoizé } \\
\text { (Don } \\
\text { patriotique) }\end{array}$ & $\begin{array}{l}\text { Renvoizé } \\
\text { (Section } \\
\text { correspondance) }\end{array}$ \\
\hline $\begin{array}{l}\text { Lepage } \\
\text { (B. } \\
\text { correspondance) }\end{array}$ & $\begin{array}{l}\text { Lepage } \\
\text { (B. correspondance, } \\
\text { renvois et scrutins) }\end{array}$ & & & \\
\hline $\begin{array}{l}\text { Atrux } \\
\text { (B. renvois) }\end{array}$ & $\begin{array}{l}\text { Atrux } \\
\text { (B. correspondance, } \\
\text { renvois et scrutins) }\end{array}$ & $\begin{array}{l}\text { Atrux } \\
\text { (Secrétariat de } \\
\text { l'Assemblée) }\end{array}$ & $\begin{array}{l}\text { Atrux } \\
\text { (Don } \\
\text { patriotique) }\end{array}$ & $\begin{array}{l}\text { Atrux } \\
\text { (Section pétitions) }\end{array}$ \\
\hline $\begin{array}{l}\text { G. Vaillant } \\
\text { (B. renvois) }\end{array}$ & $\begin{array}{l}\text { G. Vaillant } \\
\text { (B. correspondance, } \\
\text { renvois et scrutins) }\end{array}$ & $\begin{array}{l}\text { G. Vaillant } \\
\text { (Secrétariat de } \\
\text { l'Assemblée) }\end{array}$ & $\begin{array}{l}\text { G. Vaillant } \\
\text { (Don } \\
\text { patriotique) }\end{array}$ & $\begin{array}{l}\text { G. Vaillant } \\
\text { (Section pétitions) }\end{array}$ \\
\hline $\begin{array}{l}\text { Le Harivel } \\
\text { (B. renvois) }\end{array}$ & $\begin{array}{l}\text { Le Harivel } \\
\text { (B. correspondance, } \\
\text { renvois et scrutins) }\end{array}$ & $\begin{array}{l}\text { Le Harivel } \\
\text { (Secrétariat de } \\
\text { l'Assemblée) }\end{array}$ & & $\begin{array}{l}\text { Le Harivel } \\
\text { (Section } \\
\text { correspondance) }\end{array}$ \\
\hline $\begin{array}{l}\text { Baboin } \\
\text { (B. renvois) }\end{array}$ & & & & \\
\hline $\begin{array}{l}\text { Henri } \\
\text { (B. renvois) }\end{array}$ & $\begin{array}{l}\text { Henri } \\
\text { (B. correspondance, } \\
\text { renvois et scrutins) }\end{array}$ & $\begin{array}{l}\text { Henri } \\
\text { (Secrétariat de } \\
\text { l'Assemblée) }\end{array}$ & $\begin{array}{l}\text { Henri } \\
\text { (Don } \\
\text { patriotique) }\end{array}$ & $\begin{array}{l}\text { Henri } \\
\text { (Section pétitions) }\end{array}$ \\
\hline
\end{tabular}

31 À part les secrétaires-commis qui composaient la Section de correspondance - on ignore encore actuellement pour la plupart d'entre eux leur trajectoire antérieure au mois de novembre 1792 -, les autres secrétaires-commis présents dans le Comité des pétitions et de correspondance avaient une longue expérience en gestion de la correspondance. Il suffit d'observer la distribution qui était faite de ceux-ci entre les différentes sections pour voir comment on tentait de ne pas les éloigner trop des tâches qu'ils avaient exercées depuis le début du processus révolutionnaire.

Il est difficile d'évaluer jusqu'à quel point les secrétaires-commis du Comité des pétitions et de correspondance étaient au courant de toutes les affaires gérées par les assemblées parlementaires depuis le mois de juillet 1789, mais on ne peut pas sous-estimer que 11 des 20 secrétaires-commis qui en faisaient partie avaient géré la majorité des informations reçues par elles. 
33 À cet égard, on ne doit pas oublier que M. Aubusson avait fait le tri de toute la correspondance adressée directement à l'Assemblée, que MM. Atrux, G. Vaillant et Henri avaient analysé, par ordre de dates et de numéros, les pièces sans nombre qui arrivaient journellement de toutes les parties du royaume, que MM. Renvoizé et Le Harivel tenaient les registres de correspondance depuis l'été $1789^{29}$ ou qu'entre les mains de MM. Garnier, Hussenet, Chaulay, Dupuis et Chachoin était passée la majorité de la correspondance délicate qui arrivait au corps législatif, à savoir : les pétitions, les mémoires et les adresses que les commettants adressaient à l'Assemblée, non renvoyés par M. Aubusson à aucun comité en particulier ; et la correspondance relative à l'administration et à la police, c'està-dire toutes les lettres qui rendaient compte des désordres qui avaient lieu partout en France.

Toutes proportions gardées, on pourrait dire que, au mois de novembre 1792, les cinq personnes les mieux informées quant aux conflits qui avaient lieu partout en France depuis l'été 1789 étaient MM. Garnier, Hussenet, Chaulay, Dupuis et Chachoin. Et ce, non seulement parce qu'ils avaient été les secrétaires-commis du Comité des rapports sous l'Assemblée nationale constituante et du Comité des pétitions sous l'Assemblée législative mais aussi parce qu'ils l'avaient été de deux commissions de plus ${ }^{30}$ : la Commission des douze, créée le 6 mars 1792 pour penser les mesures les plus appropriées pour le maintien de l'ordre et de la tranquillité publique ${ }^{31}$; et la Commission extraordinaire des douze, établie le 17 juin 1792 pour proposer les moyens nécessaires pour sauver la Constitution, la liberté et l'Empire ${ }^{32}$. Comme nous l'avons déjà dit, les députés chargés de penser les mesures pour protéger le processus révolutionnaire pouvaient changer, être d'une tendance politique ou d'une autre, comme cela se produisit, mais les secrétaires-commis qui géraient la correspondance qui rendait compte des désordres étaient les mêmes depuis l'été 1789.

\section{Pouvoir et information : le Comité de salut public}

Le 6 avril 1793, la Convention nationale créa le très célèbre Comité de salut public afin, d'une part, de surveiller et d'accélérer l'action de l'administration confiée au Conseil exécutif provisoire, et, d'autre part, de prendre, dans des circonstances urgentes, les mesures de défense générale extérieure et intérieure nécessaires pour le maintien de l'ordre - établi par l'Assemblée - et de la tranquillité publique ${ }^{33}$. Le lendemain de cet établissement, le système de gestion d'information mit sur pied par la Convention nationale avec un projet de décret présenté par Gossuin fut entièrement modifié.

Depuis le 11 novembre 1792 le Comité des pétitions et de correspondance gérait toute la correspondance reçue par l'Assemblée: les lettres des commissaires aux armées, des ministres et des généraux, ainsi que les adresses, les pétitions et les lettres renvoyées par les commettants. Depuis le 8 avril 1793, tout allait se passer autrement, afin de connecter les flux d'informations entrantes avec le Comité de salut public.

Tout d'abord, le 7 avril 1793, le Comité de salut public avait adopté ce qui suit :

Il a été arrêté d'organiser le Comité de salut public en la manière suivante : $1^{\text {o }} \mathrm{Un}$ bureau de correspondance avec les divers commissaires de la Convention nationale près les armées et dans les départements. $2^{\circ}$ Un bureau de correspondance avec les ministres et les généraux. 3ํㅡㄴ bureau central chargé d'enregistrer les adresses, pétitions et mémoires, correspondance générale, réclamations contre le conseil exécutif provisoire et de la distribution de toutes les pièces ${ }^{34}$. 
Ensuite, le 8 avril 1793 la Convention nationale décida d'établir une correspondance directe entre le Comité de salut public et les commissaires aux armées. Jusqu'à ce moment-là, en effet, cette correspondance était assurée par la section de correspondance du Comité des pétitions et de correspondance.

Article $1^{\mathrm{er}}$ : Tous les commissaires de la Convention nationale près les armées, dans les départements frontières, maritimes et de l'intérieur, entretiendront avec le Comité de salut public une correspondance journalière, indépendamment de leur correspondance avec la Convention nationale ${ }^{35}$.

En outre, le même 8 avril, les députés décidèrent d'établir une Commission des dépêches pour fournir au Comité de salut public toute l'information importante qui pouvait arriver au corps législatif. Une intervention faite le 29 avril 1793 par un membre de la Commission des dépêches ainsi le confirmait :

Un membre de la Commission des dépêches observe à la Convention nationale : $1^{\circ}$ Qu'elle a décrété le 8 de ce mois l'établissement d'une commission chargée spécialement de l'examen des lettres des Ministres, des Généraux, de ses Députés dans les départements et des corps administratifs; de donner la lecture, soit en totalité, soit par extrait de ces différentes pièces et de renvoyer au Comité de salut public les pièces dont la lecture ne pourrait être donnée sans inconvénient ${ }^{36}$.

Or, la Convention nationale non seulement avait créé la Commission des dépêches pour décider de ce qui pouvait être lu à l'Assemblée et de ce qui devait être renvoyé au Comité de salut public, et pour examiner les lettres des ministres, des généraux, des commissaires envoyées en missions et des corps administratifs, elle l'avait établie également pour gérer toute la correspondance qui parvenait au corps législatif :

$2^{\circ}$ Que la Convention a décrété le 10 que la Commission des dépêches est autorisée à renvoyer aux divers comités les lettres et pièces énoncées qui n'ont qu'un objet particulier et sur lesquelles elle ne peut statuer sans un rapport ${ }^{37}$.

Seulement deux jours après la création du Comité de salut public, le Comité des pétitions et de correspondance perdit la compétence de gérer la correspondance des députés envoyés en mission et de distribuer lui-même la correspondance reçue par le corps législatif. C'est-à-dire qu'il perdit le contrôle du Bureau des renvois et de correspondance reconverti en sa deuxième section.

L'établissement du Comité de salut public ayant en son sein une majorité montagnarde ${ }^{38}$ enleva au Comité des pétitions et de correspondance, qui n'en avait pas ${ }^{39}$, son statut de " centre de toutes les affaires de la Convention nationale.»

Avec l'établissement de la Commission des dépêches on évita que les informations qui devaient passer au Comité de salut public fussent analysées d'abord par un comité dont les membres les plus anciens sortaient des rangs de la Plaine. Au début du mois d'avril 1793, Constant Joseph Eugène Gossuin (plaine), Jean Sautereau (plaine) et Hector Soubeyrand de Saint Prix (girondin), membres du Comité des pétitions et de correspondance, travaillaient depuis le mois d'octobre 1791 dans le Comité des pétitions. Alors que Jean Baptiste Benoît Monestier (montagnard), Jacques Paul Fronton Duplantier (girondin), Claude Alexandre Ysabeau (montagnard), Claude François Bruno Siblot (plaine), Anne Alexandre Marie Thibault (plaine), Yves Marie Audrein (plaine), Pierre Paganel (plaine) et Jean Ducos (girondin) travaillaient dans le Comité des pétitions et de correspondance depuis le mois d'octobre 1792. Des onze députés qui travaillaient depuis le plus longtemps dans ce Comité, deux étaient montagnards, trois étaient girondins et six étaient de la plaine. D'autre part, avec l'établissement de la Commission des dépêches, 
on évita également que la première lecture des lettres des ministres, des généraux, des députés envoyés en mission et des corps administratifs, pouvaient être faite par un comité qui, au début du mois d'avril 1793, après deux rénovations, était composé de quatre députés girondins, dix députés de la plaine et dix députés montagnards.

Tout semble indiquer que les compétences attribuées à la section dans laquelle travaillait M. Aubusson étaient trop importantes pour : d'une part, les laisser en marge du Comité de salut public ; et, d'autre part, les laisser aux mains d'un groupe de députés dans lequel les montagnards n'étaient pas majorité. S'il n'en était pas ainsi, pourquoi créer une commission pour effectuer un travail que faisait déjà la Section des renvois et dons patriotiques sous les ordres du Comité des pétitions et de correspondance? Pourquoi ne pas décréter simplement que cette section devait renvoyer au Comité de salut public les informations importantes ou délicates?

\section{La Commission des dépêches : un tremplin par M. Aubusson ?}

La Commission des dépêches créée par la Convention nationale retira au Comité des pétitions et de correspondance quelque chose de plus que les compétences gérées par l'ancien Bureau des renvois et de correspondance, reconverti en Section des renvois et dons patriotiques le 21 novembre 1792 : elle lui retira MM. Aubusson et Thibault, tous les deux chargés de distribuer la correspondance reçue sous la Première République.

Comme cela s'était produit au mois de novembre 1792, les députés remplacèrent les individus (les députés) qui pouvaient gérer les compétences de l'ancien Bureau des renvois et de correspondance mais ne changèrent pas les individus (les secrétairescommis) qui y travaillaient; de telle manière que, lorsque la Commission des dépêches fut mise sur pied en accomplissement du décret du 8 avril, M. Aubusson fut appelé à nouveau pour s'occuper de la gestion de la correspondance reçue.

De fait, dès le lendemain de la création de cette nouvelle commission, MM. Aubusson et Thibault en faisaient déjà partie ${ }^{40}$. Cependant, ils ne furent pas les seuls à y travailler. MM. Levasseur, Christiani, Rudel, Périès et Guyardin, membres de la Commission des dépêches, considérant qu'ils nécessitaient un secrétaire-commis supplémentaire, décidèrent de nommer comme troisième secrétaire-commis de la commission $\mathrm{M}$. Cheron ${ }^{41}$ qui travaillait dans le corps législatif depuis le début du processus révolutionnaire, adressant aux comités et aux députés les lettres qu'ils devaient recevoir et recueillant celles que ceux-ci voulaient envoyer par la petite poste. Selon un rapport présenté à l'Assemblée nationale constituante le 28 août 1791 :

Le sieur Charon prend tous les jours au Bureau de correspondance les lettres et paquets envoyés aux comités de l'Assemblée et à MM. les députés sous le couvert de M. le président. Il met et rectifie les adresses, et fait parvenir aux comités et à MM. les députés les lettres et paquets qui leur sont destinés. Il reçoit aussi toutes les lettres que l'Assemblée, ses comités et MM. les députés veulent envoyer par la petite poste, les contresigne et les expédie ${ }^{42}$.

Sous la direction de deux députés montagnards (Levasseur et Guyardin), d'un député modéré (Rudel), d'un député de la plaine (Christiani) et d'un député girondin (Périès), M. Aubusson gravit les échelons au sein de la Commission des dépêches ${ }^{43}$. Le 28 juin 1793, seulement deux mois et demi après son entrée dans cette commission, on lui donna la responsabilité de surveiller toute la correspondance qui circulait au sein de la 
Commission des dépêches, de même que celle de surveiller les travaux de ses confrères. De fait, on le nomma chef du bureau :

Nous députés à la Convention Nationale membres de la Commission des Dépêches, ayant reconnu qu'il est indispensable pour le bien et la célérité du service, de confier à l'un des secrétaires commis attachés à notre bureau, connu pour son patriotisme, son intelligence et son exactitude (sous sa responsabilité personnelle) le soin et la garde de toutes les pièces et la surveillance du travail de ses confrères, avons nommé pour occuper la place sus dite, en qualité de chef, le citoyen Aubusson, employé dans les bureaux des Assemblée Constituante et Législative, et de la Convention Nationale depuis le premier juillet mil sept-cents quatre-vingtneuf, et qui nous a paru réunir les qualités requises pour bien remplir les fonctions importantes que nous jugions nécessaires de lui déléguer. Fait et arrêté à Paris le vingt-huit juin mil sept cent quatre-vingt-treize l'an 2 de la République une et indivisible ${ }^{44}$.

Par ailleurs, quatre mois et une semaine seulement après avoir commencé à travailler à la Commission des dépêches, son salaire fut augmenté pour la première fois. Il cessa de toucher 1800 livres l'an pour en toucher $2400^{45}$. Ses compétences - intelligence, exactitude et patriotisme - semblaient être revues à la hausse.

\section{Dons et serments de fidélité : le patriotisme d'un secrétaire- commis}

Le 20 avril 1792, l'Assemblée législative déclara la guerre au roi de Bohème et de Hongrie. Deux jours plus tard, le président de l'Assemblée lisait une lettre signée de M. Aubusson et de ses compagnons, MM. Lepage le jeune, Henri, G. Vaillant, Renvoizé, Atrux, Férès, Le Harivel et Devilliers - tous secrétaires-commis du Bureau des renvois et de correspondance, à l'exception du dernier qui travaillait au Bureau de recensement des scrutins, de la distribution des billets, de l'expédition des passeports - dans laquelle ils déclaraient faire un don de 540 livres par an pendant la durée de la guerre ${ }^{46}$. M. Aubusson et ses confrères décidaient volontairement de donner 60 livres l'an de leur salaire pour contribuer aux frais de la guerre. Cela signifiait, pour chacun d'entre eux, 5 livres par mois; une quantité non négligeable que l'Assemblée en remerciement décida de mentionner dans son procès-verbal.

51 Selon les Archives parlementaires, le 4 juillet 1792, M. Aubusson et ses collègues déposèrent en assignats les 5 livres auxquels ils s'étaient engagés le 22 avril ; de même que le firent ce même jour les secrétaires-commis du Comité de finances et du Comité de commerce ${ }^{47}$. D'autres secrétaires-commis à contribuer aux frais de la guerre furent ceux du Comité des

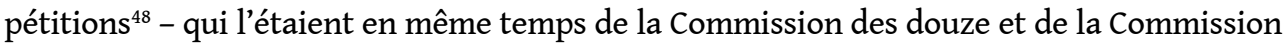
extraordinaire des douze -qui le firent à plusieurs reprises, et ceux du Comité de commerce $^{49}$ et ceux du Comité de division ${ }^{50}$.

Par ailleurs, le 15 août 1792, cinq jours après la chute de la monarchie, l'Assemblée décréta que « tous les fonctionnaires publics [étaient] tenus de prêter, dans la huitaine du jour de la publication du présent décret, le serment d'être fidèles à la nation et de maintenir de tout leur pouvoir la liberté et l'égalité, ou de mourir à leur poste ${ }^{51}$.» Quelques instants après l'adoption de ce décret, M. Aubusson et ses confrères des différents bureaux du Secrétariat de l'Assemblée se présentèrent devant des députés pour prêter le serment. Le président de l'Assemblée applaudit leur civisme et leur accorda les honneurs de la séance ${ }^{52}$. 

poursuivirent, donnant des preuves de leur engagement révolutionnaire. Le 5 novembre 1792, alors qu'ils n'étaient pas encore membres du Comité des pétitions et de correspondance, et qu'ils se trouvaient sous les ordres du président de l'Assemblée, ils firent le don correspondant au mois d'octobre. Cette fois-là, ce n'était plus les mêmes neuf secrétaires-commis du mois d'avril. MM. Lepage, Le Harivel, Férès et Devilliers ne firent pas leur don. Cependant, deux nouveaux secrétaires-commis vinrent s'ajouter à l'initiative : il s'agissait de MM. Thibault et Rimonneau ${ }^{53}$.

Enfin, le dernier don localisé de M. Aubusson date du 5 mars 1793, quelques semaines avant qu'il ne commençât à faire partie de la Commission des dépêches. Cette fois-ci, il fit seul son don et déposa les assignats correspondant aux mois de décembre 1792 et janvier et février $1793^{54}$. On ignore encore le montant total que M. Aubusson finit par donner pour contribuer aux frais de la guerre mais, en tenant compte des données localisées jusqu'à ce moment, on sait que du mois d'avril 1792 au mois de février 1793, M. Aubusson donna 55 livres. Avec tout cela, peut-on y voir une pratique de distinction, une tactique pour obtenir une fonction plus élevée dans l'Assemblée ? On ne le sait pas, mais dans tous les cas, cela lui permit d'obtenir une sorte de « brevet de patriotisme ${ }^{55}$.»

\section{Monsieur Aubusson : le secrétaire principal du Comité de salut public}

Le jour même où le Comité de salut public reçut la commande de présenter les noms de ceux qui devaient composer les différents comités de la Convention nationale ${ }^{56}$, le 13 septembre 1793, celui-ci nomma M. Aubusson comme secrétaire principal de ses différentes sections :

Le Comité de salut public, vu les travaux urgents et multipliés de ses bureaux, arrête que le citoyen André Aubusson, chef du Bureau de la commission des dépêches de la Convention nationale, cessera provisoirement ses fonctions dans ce bureau, après s'être concerté avec les députés membres de la commission des dépêches, et qu'il prendra, à compter de ce jour, les fonctions de secrétaire principal du comité, et qu'il lui sera accordé une indemnité de 6000 livres l'année ${ }^{57}$.

Après cinq mois et une semaine de travail dans la Commission des dépêches, M. Aubusson vint travailler sous les ordres du Comité de salut public et reçut à cet effet un salaire annuel de 6000 livres. Pour la première fois, il abandonnait le poste qu'il occupait depuis le début de la Révolution. À partir de ce moment-là, M. Thibault le remplaça dans sa charge au sein de la Commission des dépêches, après deux mois et demi comme chef de ce bureau et après avoir été durant quatre ans et deux mois le principal responsable de la distribution de toute la correspondance adressée au corps législatif.

Ses compétences au sein du Comité de salut public n'en furent pas moins importantes. En effet, on l'avait appelé pour coordonner les travaux des sept bureaux qui composaient, à la fin de l'été 1793, le grand Comité de salut public. À savoir : le Bureau central, le Bureau d'enregistrement, le Bureau de la correspondance pour les ministres et les commissaires, le Bureau de la correspondance du corps administratif et des sociétés populaires, le Bureau de la correspondance de la guerre et de la marine, le Bureau pour les manufactures d'armes et le Bureau des députations ${ }^{58}$; soit sept bureaux au total pour la gestion desquels on lui octroyait un salaire mensuel de 500 livres $^{59}$. C'était le même montant que recevait le directeur général du Comité de sûreté générale ou que pouvait 
recevoir un chef de bureau du Ministère de l'Intérieur. Ce montant était bien supérieur aux 25 ou 100 livres mensuelles que pouvait toucher le personnel moins qualifié du Comité de salut public, c'est-à-dire les garçons de bureau et assimilés ${ }^{60}$.

D'autre part, on sait que l'arrêté qui nommait M. Aubusson comme secrétaire principal du Comité de salut public portait les signatures de Claude-Antoine Prieur-Duvernois, Thuriot et Carnot; mais on ne connaît pas encore les raisons qui conduisirent ces trois députés à le nommer. Ils auraient pu promouvoir un des chefs de bureau qui travaillaient déjà dans ce comité. Également, on ne sait pas si les membres du Comité de salut public appréciaient les travaux que M. Aubusson effectuait au sein de la Commission des dépêches, s'ils le connaissaient personnellement ou si quelqu'un de la Commission des dépêches leur avait loué ses compétences ; à savoir, par exemple, Joseph Le Bon, membre de la Commission des dépêches et, à ce qu'il semble, oreille de Robespierre au sein du Comité de sûreté générale ${ }^{61}$. Or, tout paraît indiquer que M. Aubusson pouvait être le secrétaire de grand mérite que Robespierre souhaitait.

\section{Le désir de Maximilien Robespierre : un secrétaire de grand mérite}

Maximilien Robespierre ne fut pas parmi les premiers députés à entrer dans le Comité de salut public; en fait, il fut le vingt-quatrième ${ }^{62}$. Et quand finalement il le fit, le 27 juillet 1793, exactement un an avant sa détention, environ trois mois et demi s'étaient écoulés depuis sa création ${ }^{63}$. Par conséquent, au moment d'y entrer, il connaissait bien l'organisme, ses missions et ses compétences, le caractère et la pensée politique de ses membres ainsi que le contenu de ses travaux. Or, il discutait et critiquait ouvertement ces derniers, s'il lui semblait pertinent de le faire afin de les améliorer.

Robespierre connaissait en outre quelque chose de plus du Comité de salut public: sa fragilité. Malgré l'importance de ses missions et de ses compétences, le Comité de salut public était un comité provisoire, qui devait demander chaque mois aux députés de la Convention nationale l'autorisation pour poursuivre ses activités. C'était un comité fortement menacé depuis ses débuts. Personne, en effet, n'hésitait à critiquer ses opérations ni même à les censurer directement. C'est ainsi que Robespierre, à partir du mois de juin 1793, alors qu'il n'était pas encore membre de ce comité, commença à le défendre des critiques qu'il recevait, bien que lui-même l'eût critiqué durement en certaines occasions. Il le fit notamment le 14 juin 1793 au Club des Jacobins :

Il est des moments où j'ai jugé sévèrement ce comité ; mais après un sérieux examen, je me suis convaincu que ce comité désirait sincèrement le salut de la République, et il est impossible que des hommes occupés d'intérêts aussi pressants que multipliés ne soient pas exposés à des surprises, il faut les juger pour l'ensemble de leurs travaux et non pas pour leurs opérations partielles. Ne croyez pas que je prêche le modérantisme, au contraire, je prêche la surveillance la plus rigoureuse ${ }^{64}$.

Il le fit aussi le 8 juillet de la même année :

Il a fait des fautes sans doute. Est-ce à moi de les dissimuler? Pencherai-je vers l'indulgence, moi qui crois qu'on n'a point assez fait pour la patrie, quand on n'a pas tout fait, moi qui suis persuadé que la liberté récompense abondamment de ses sacrifices l'homme qui n'a plus rien à lui sacrifier ? Oui, il a fait des fautes, et je veux les lui reprocher avec vous. Mais, il y a loin des torts qu'on peut reprocher à quelqu'uns de ses membres, à la proscription dont on veut l'envelopper en masse. Oui, il est dans son sein des membres purs, irréprochables... et ce matin même, l'un 
d'eux ne nous a-t-il pas fait un rapport qui, quoiqu'il laissât quelque chose à désirer encore, n'en portoit pas moins l'empreinte du grand talent et de l'âme vraiment républicaine de son auteur. En un mot, je soutiens qu'il seroit fort impolitique, en ce moment, d'appeler la défaveur du peuple sur un comité qui a besoin d'être investi de toute sa confiance; qui est chargé de grands intérêts, et dont la patrie attend de grands secours; et, quoiqu'il n'ait pas l'agrément des citoyennes républicaines révolutionnaires, je ne le crois pas moins propre à ses importantes opérations ${ }^{65}$.

Le 10 juillet Pierre Joseph Cambon, Louis Bernard Guyton-Morveau, Georges Jacques Danton, Jean François Bertrand Delmas, Jean François Delacroix, Dominique Vincent Ramel-Nogaret, Théophile Berlier et François René Auguste Mallarmé quittèrent le Comité de salut public. Dix-sept jours plus tard, Maximilien Robespierre y entra, avec des idées tout à fait précises sur la forme que devait adopter cette institution provisoire et révolutionnaire. De ce point de vue, le $1^{\mathrm{er}}$ août 1793, Robespierre se montra clairement très défavorable à la proposition de Danton d'établir ce comité en gouvernement provisoire, surtout sans un projet préalable de réorganisation :

$\mathrm{Si}$, en changeant l'état du gouvernement actuel, on y substituait un état certain et stable, j'appuyerais la proposition de Danton; mais en détruisant l'autorité du Conseil exécutif, je ne vois pas qu'on fasse mieux marcher le gouvernement. La proposition me paraît vague, son objet d'une exécution incertaine. Il serait dangereux de paralyser subitement le gouvernement existant. Il ne suffit pas de dire : il n'y aura plus de Conseil exécutif, et le Comité de salut public sera un gouvernement provisoire; il faut organiser ce gouvernement, et comme on ne nous en a pas encore montré les moyens, je demande l'ajournement de la proposition ${ }^{66}$.

Ensuite, le 29 août 1793, il se montra nettement contraire à la création de commissions qui pourraient mettre en danger les compétences du Comité de salut public. Il le manifesta notamment après une intervention de Billaud Varenne visant à créer une commission pour surveiller les ministres :

J'observe que la commission que vous instituerez pour surveiller les ministres pourrait donner un résultat différent que celui que vous attendez; car il est à craindre que cette commission ne s'occupe plutôt d'inimités personnelles que de surveillance loyale, et ne devienne ainsi un véritable Comité de dénonciations (Violents murmures). Citoyens, je dois dire avec franchise que ce n'est pas d'aujourd'hui que je m'aperçois qu'il existe un système perfide visant à paralyser le Comité de salut public en paraissant l'aider dans ses travaux, et qu'on cherche à avilir le pouvoir exécutif, afin qu'on puisse dire qu'il n'y a plus en France d'autorité capable de manier les rênes du gouvernement. Si vous créez cette Commission, vous entravez la marche du Comité de salut public, et vous allez contre le but que vous vous proposez. Je demande la question préalable sur la proposition de Billaud ${ }^{67}$.

63 À la fin du mois d'août 1793, la survie du Comité de salut public continuait à être menacée, de même que ses compétences. Sa fragilité était réelle et les interventions de Robespierre permettent d'observer comment celui-ci tentait d'en protéger les compétences. Et ce, non seulement face à la création d'une nouvelle commission qui pourrait intervenir dans ses travaux sinon aussi face au Comité de sûreté générale. Le 25 août 1793, au Club des Jacobins, Robespierre demanda de limiter les compétences de ce dernier comité, de le renouveler et de le réduire à dix membres :

Le Comité de salut public, chargé de déjouer les complots de quelque nature qu'ils soient, fait souvent arrêter telle personne que le Comité de sûreté générale dont les fonctions sont à peu près les mêmes avait fait rechercher aussi. Il en résulte un conflit de juridiction qui devient souvent nuisible au salut de l'État. Le Comité de sûreté générale fait mettre en liberté ou absout celui que le Comité de salut public 
avait incarcéré ou condamné, parce que tous les deux ne jugent pas sur les mêmes pièces, et qu'on supprime avec l'un ce qui a servi de base aux jugements de l'autre, ce qui laisse toujours une porte à l'intrigue. Je me résume : [...] renouvellement du Comité de sûreté générale et sa composition de dix membres seulement, dont les fonctions seront déterminées et on tirera une ligne de démarcation entre ses fonctions et celles du Comité de salut public ${ }^{68}$. les critiques et les dangers qui le menaçaient. Il élabora également, dans une note de quatorze points, comment devait être organisé ce comité afin d'opérer avec tous les travaux qu'il devait effectuer :

Il faut : Un secrétaire général de grand mérite. Un bureau de secrétaires particuliers intelligents et patriotes. Des agents également sûrs pour transmettre les arrêtés du Comité à ceux qui doivent les exécuter. Il faut que dans les 24 heures ceux qui sont chargés de l'exécution en rendent compte au Comité. Il faut déterminer à qui seront confiés les ordres d'arrêtés et par qui ils seront transmis. Il faut des commis très sûrs attachés au Comité. Il faut que chaque membre du Comité ait une tâche particulière, qu'il soit entouré de secrétaires et d'agents dignes de confiance. Il faut que chaque membre ait un emplacement séparé où il puisse travailler, et toutes les commodités physiques nécessaires pour agir. Il faut que le Comité nomme un commissaire pour entendre les députations et les demandes particulières. Il faut que le Comité nomme des rapporteurs pour les affaires particulières. Que le Comité soit fermé et inaccessible, hors les cas très extraordinaires, et qu'il ait des agents pour maintenir cette partie de sa police. Que le Comité ne délibère jamais en présence d'aucun étranger. Que les heures des séances soient rigoureusement fixées. Que les noms de tous les agents du Comité soient remis à chacun de ses membres ${ }^{69}$.

Dans le premier point qu'il rédigea, il indiquait que ce qui convenait au Comité de salut public était un secrétaire général de grand mérite. M. Aubusson, l'homme qui savait tout, était-il le secrétaire général de grand mérite que Robespierre souhaitait pour ce Comité ?

On ignore le moment auquel Robespierre écrivit cette note, cependant, on sait parfaitement que, le 13 septembre 1793, M. Aubusson, chef des secrétaires-commis de la Commission des dépêches, fut appelé par le Comité de salut public pour devenir son secrétaire principal, et que Robespierre, présent à la réunion du Comité de salut public, adopta l'arrêté lui donnant la charge de secrétaire principal que Claude Antoine PrieurDuvernois, Thuriot et Carnot avaient signé.

\section{Le secrétaire personnel de Guyton-Morveau : le deuxième secrétaire principal du Comité de salut public}

M. Aubusson continua de travailler au Comité de salut public comme secrétaire principal jusqu'au dernier jour de la Convention nationale. Toutefois, il ne fut pas longtemps le seul secrétaire principal de ce Comité. Une semaine après avoir obtenu la charge, le Comité de salut public décida de nommer un second secrétaire principal : un certain J. B. L. Pierre, né à Dijon ${ }^{70}$, et chef du Bureau d'enregistrement de ce Comité. Le Comité de salut public considéra que M. Aubusson ne pouvait mener à terme seul les compétences accordées et il adopta donc l'arrêté suivant :

Le Comité de salut public, vu l'urgence et la multiplicité du travail de ses bureaux, considérant que le secrétaire principal ne peut suffire seul à une surveillance et à des détails aussi étendus, arrête que le citoyen Pierre, l'un des chefs de ses bureaux, 
sera adjoint dès ce jour au citoyen Aubusson, secrétaire principal, et qu'il lui sera accordé une indemnité de 5000 livres l'année ${ }^{71}$. mais ce que l'on sait c'est que les compétences de M. Pierre était fortement appréciées par Louis Bernard Guyton-Morveau - nommé le 7 avril 1793 pour faire partie du premier Comité de salut public - et que la trajectoire de celui-ci pour devenir second secrétaire principal du Comité de salut public avait été fort différente de celle suivie par M. Aubusson. Une lettre écrite par M. Pierre le 6 prairial an IV racontait sa trajectoire ${ }^{72}$. De 1789 à 1790 en partie il avait été employé au greffe de la mairie de la municipalité de Dijon. De 1790 en partie jusqu'à 1791 il avait été employé au commissariat de Dijon de la ci-devant province de Bourgogne. En 1791 il était devenu secrétaire du citoyen GuytonMorveau, procureur général syndic du Département de la Côte d'Or. À partir du mois de septembre 1791 il était devenu secrétaire du même citoyen Guyton-Morveau, d'abord, représentant du peuple à l'Assemblée législative, ensuite, à la Convention nationale. Finalement, depuis l'établissement du Comité de salut public - qui comptait avec la présence de Guyton-Morveau - et jusqu'à sa dissolution, il avait été chef du Secrétariat, secrétaire principal et secrétaire général de ce comité. D’abord, avec une assignation de 5000 livres par an, mais à partir du mois de germinal an II, avec une assignation de 6000 livres par an. La même que celle que M. Aubusson avait depuis son entrée au Comité de salut public comme secrétaire principal.

Même si les trajectoires de ces deux secrétaires étaient bien distinctes - M. Aubusson gravit des positions grâce à son travail dans les différents bureaux qu'il y eût pour gérer la correspondance reçue ${ }^{73}$, et $\mathrm{M}$. Pierre les gravit grâce à son étroite relation professionnelle avec le député Guyton-Morveau - les deux secrétaires travaillèrent ensemble jusqu'à la cessation des fonctions du Comité de salut public le 13 brumaire an IV 74. À partir de cette date et jusqu'aux derniers mois du Directoire, ils continuèrent à travailler côte à côte mais, cette fois-ci, au Secrétariat général du Directoire.

\section{M. Aubusson : chef de la Division centrale du Directoire exécutif}

71 Après être demeuré à Versailles pendant les premiers mois de l'Assemblée nationale constituante, et avoir résidé ensuite à Paris à partir du mois d'octobre 1789 - rue Jacob du Faubourg Saint-Germain, rue des Moulins, rue des Moineaux, rue Favart et enfin rue des Fossoyeurs no $1045^{75}-$ M. Aubusson était depuis le 13 brumaire an IV le chef de la Division centrale du Directoire exécutif. Son expérience en tant que secrétaire principal du Comité de salut public lui avait ouvert les portes - à lui-même ainsi qu'à M. Pierre - des bureaux du Directoire, le nouveau pouvoir exécutif à partir du mois d'octobre1795. Leur expérience était bien appréciée tout autant que leur engagement révolutionnaire et leur attachement à la Constitution de 1795.

72 Suite à la réorganisation du Secrétariat général du Directoire le 4 nivôse an $\mathrm{V}$ (24 décembre 1796), M. Aubusson écrivit une lettre dans laquelle il louait son engagement 
révolutionnaire en soulignant son attachement à la Révolution lors des fameuses journées de thermidor an II (chute de Robespierre), prairial an III (fin des derniers Montagnards) et vendémiaire an IV (insurrection royaliste) ${ }^{76}$. Dans cette lettre, il expliquait qu'il n'avait jamais abandonné son poste pendant un instant; qu'il n'avait jamais été flétri par un soupçon ; qu'il avait la conviction intime d'avoir rempli en toutes occasions avec courage, zèle et énergie les devoirs d'un bon patriote et d'un vrai républicain étranger à tous les partis; qu'il n'avait jamais vu que la chose publique, sans nulle espèce d'ambition; qu'il avait dédaigné les places brillantes que tant d'autres convoitaient avec ardeur; et qu'il n'avait jamais désiré que vivre honorablement du fruit de son travail. À la fin de sa lettre, il jurait de défendre la Constitution de 1795 et de tenir ce serment au prix de sa vie.

À l'égard aussi de cette réorganisation, $\mathrm{M}$. Aubusson adressa le 6 nivôse an $\mathrm{V}$ au citoyen La Garde, secrétaire général du Directoire exécutif, une lettre pour obtenir la commission de son collègue M. Pierre, de la sienne et de celle de ses collaborateurs et des garçons de bureau $^{77}$. Tous les employés de sa division lui avaient écrit en vertu d'un arrêté adopté le $1^{\mathrm{er}}$ nivôse pour lui demander son agrément pour l'obtention de leur commission. C'est le cas, par exemple, des citoyens Mignot et Lejolliot :

Citoyen. Conformément au règlement de l'organisation définitive du Secrétariat général du Directoire, je vous prie d'accorder votre agrément pour l'obtention de la commission qui m'est nécessaire pour continuer mes travaux. Salut et Fraternité. Mignot ${ }^{78}$.

Au citoyen Aubusson chef de la division centrale du Secrétariat général du Directoire exécutif. Je dois aux termes du règlement d'organisation obtenir ton agrément par écrit pour avoir du citoyen Lagarde ma commission de sous-chef de la division centrale. Je viens avec confiance t'en faire la demande. Mes anciens services et continus, ta justice et ton amitié semblent m'assurer ton suffrage ${ }^{79}$.

Tout au long du Directoire, M. Aubusson fut chef de la Division centrale du Secrétariat général du Directoire exécutif mais, exceptionnellement, il substitua M. La Garde comme secrétaire général du Directoire exécutif en deux occasions. Il le substitua le 11 août 1798 ${ }^{80}$ quand M. La Garde tomba malade; et aussi le 26 mai $1799^{81}$. Pendant ce temps, M. Aubusson travailla conjointement avec M. Pierre qui était chef-adjoint de la Division centrale $^{82}$. Pour ces fonctions, ils touchèrent le même salaire, dont le montant ne fut pas toujours le même. Au moment de la dissolution du Comité de salut public ils avaient un salaire de 8000 livres par an; lors d'un décret adopté le 17 frimaire an IV leurs appointements étaient de 7500 livres en assignats par mois, et quelques mois après de 12000 livres en assignats par mois ${ }^{83}$; à partir du mois de pluviôse an $\mathrm{V}$ leurs appointements étaient de 6000 francs par an et à partir du mois de frimaire an VI et jusqu'à la fin du Directoire ils étaient de 8000 francs par an ${ }^{84}$.

75 Depuis leur entrée au Secrétariat général du Directoire exécutif, MM. Aubusson et Pierre prêtèrent à plusieurs reprises serment de fidélité à la République française. Ils le firent tous les 21 janvier - jour de l'exécution de Louis XVI. Le premier serment, ils le firent après l'insurrection royaliste du 13 vendémiaire an IV ( 5 octobre 1795$)$ le $1^{\mathrm{er}}$ pluviôse an IV. Ce jour-là ils jurèrent d'être sincèrement attachés à la République française une et indivisible et de vouer une haine éternelle à la Royauté. Au cours des années suivantes, ils jurèrent haine à la Royauté et à l'anarchie, ainsi que leur attachement et fidélité à la République et à la Constitution de l'an III ${ }^{85}$.

76 Après le coup d'État de Napoléon Bonaparte, le 18 brumaire an VIII (9 novembre 1799), M. Aubusson délivra aux Archives nationales les Cahiers mensuels d'enregistrement de pièces remises à la division des procès-verbaux du Directoire pour la rédaction des procès-verbaux, 
aujourd'hui consultables sous la référence $\mathrm{AF} / \mathrm{III} / 307$ et $308^{86}$. Dix années après son entrée au Bureau de correspondance de l'Assemblée nationale constituante, il continuait encore à prêter ses services.

\section{Conclusions}

77 La première conclusion de ce travail qui a été divisé en deux parties à cause de son extension originale est l'attention donnée par les députés des différentes assemblées parlementaires, entre 1789 et 1795 , à la manière et aux personnes qui devaient gérer la correspondance reçue dans le cadre du pouvoir législatif. Pour cette raison même, ceux qui dirigeaient des postes-clés pour faire circuler les informations n'étaient pas toujours les mêmes: le président de l'Assemblée sous l'Assemblée nationale constituante et l'Assemblée législative, le Comité des pétitions et de correspondance sous les premiers mois de la Convention nationale ou la Commission des dépêches après l'établissement du Comité de salut public. Pour cette raison-là aussi, M. Aubusson vit toujours ses compétences menacées, soit parce que les députés voulaient se faire lire toute la correspondance et décider par eux-mêmes où la renvoyer soit parce que certains députés voulaient que ce travail fût effectué par un comité et pas par un bureau sous le contrôle du président de l'Assemblée, soit enfin parce qu'un nouvel organisme était créé, comme la Commission des dépêches, pour assumer le travail qui était déjà assumé par la Deuxième Section du Comité des pétitions et de correspondance où travaillait précisément $M$. Aubusson. Et tout cela, enfin, parce que les députés savaient bien que leurs décrets dépendaient dans une bonne mesure des informations dont ils avaient connaissance, mais également que le débat parlementaire pouvait être facilement orienté selon les pièces qui leur étaient communiquées.

La deuxième conclusion de ce travail est la présence d'employés de base qui demeurent pendant des années dans le bureau dans lequel ils ont commencé à travailler après les premiers moments de l'Assemblée nationale constituante; et conservent leurs fonctions au fil des années. En fait, même quand il y a des réorganisations internes dans ces bureaux, on s'occupe de respecter les fonctions des employés grâce auxquelles ils deviennent des personnels, expérimentés, spécialisés et de grand mérite. C'est le cas, par exemple, des secrétaires-commis du Comité des pétitions et de correspondance de la Convention nationale et des réorganisations opérées dans le cadre de ce Comité.

79 En outre, on n'observe pas d'épurations au moment où l'on passe de l'Assemblée nationale constituante à l'Assemblée législative, pas davantage au moment où l'on passe de l'Assemblée législative à la Convention nationale. Les secrétaires-commis qui disparaissent sont rares, et ceux qui le font, le font provisoirement ${ }^{87}$, normalement pour accompagner dans sa mission un député de leur comité nommé représentant en mission ou pour combattre dans l'armée, et toujours avec l'engagement de la part de leur comité de les admettre à nouveau à leur retour à Paris.

80 La troisième conclusion de ce travail concerne le fait qu'en suivant la trajectoire de M. Aubusson la thèse de Max Weber selon laquelle les fonctionnaires peuvent progresser dans leur carrière, grâce à leurs efforts et à leur dévouement pour exercer efficacement leurs fonctions, se confirme. Au $1^{\mathrm{er}}$ juillet $1789 \mathrm{M}$. Aubusson est un ancien militaire qui occupe un poste de secrétaire-commis dans le Bureau de correspondance; le 9 avril 1793 il entre à travailler à la Commission des dépêches et le 28 juin il devient chef de son bureau ; le 13 septembre 1793 il est nommé secrétaire principal du Comité de salut public, 
et le 13 brumaire an IV il est nommé chef de la Division centrale du Directoire exécutif, substituant le secrétaire général du Directoire exécutif en son absence.

81 La quatrième conclusion tient au fait que toutes les carrières ne se firent pas dans les bureaux des assemblées parlementaires. Le cas de M. Pierre, second secrétaire principal du Comité de salut public, est illustratif à cet égard. Il entra au Comité de salut public parce qu'il était le secrétaire privé de Guyton-Morveau. Quand ce député fut nommé membre du Comité de salut public, M. Pierre qui l'accompagnait comme secrétaire depuis 1791 entra lui aussi dans les bureaux de ce Comité. À partir de ce moment-là, en effet, l'ascension de M. Pierre se fit dans le cadre de l'administration révolutionnaire et après le 13 brumaire an IV il fut nommé chef adjoint de la Division centrale du Directoire exécutif.

À propos des carrières de MM. Aubusson et Pierre, Raphaël Matta Duvignau a souligné qu'elles n'avaient pas évolué sous le Comité de salut public ${ }^{88}$. Certes, elles n'avaient pas évolué dans le cadre de ce Comité, mais elles l'avaient fait depuis le début du processus révolutionnaire jusqu'à sa fin avec le coup d'état de Napoléon Bonaparte.

La cinquième conclusion à tirer est que les arguments soutenus par Jean René Suratteau ne se confirment pas si l'on prend en considération les trajectoires professionnelles de MM. Aubusson et Pierre :

Directeurs et ministres furent aidés par tout un monde d'employés de bureaux (6 puis 9 bureaux) qu'ils choisirent sans contrôle parmi le personnel thermidorien, souvent parmi les conventionnels non réélus ${ }^{89}$.

Ces deux trajectoires professionnelles et la continuité que l'on a observée dans le cadre des trois premières assemblées parlementaires d'un même personnel de base confirment plutôt les thèses de Catherine Kawa selon lesquelles « les commis expérimentés formaient toujours une large majorité chez les nouveaux venus, en l'an II comme en l'an V » et « les révolutionnaires n'ont pas confié à n'importe quels débutants le soin d'exécuter leur politique $^{90}$.»

85 La sixième et dernière conclusion à tirer est que les institutions ne louèrent pas seulement l'expérience et le mérite de leurs secrétaires-commis. Ils louèrent aussi leur patriotisme. À savoir: les dons patriotiques qu'ils faisaient et les serments de fidélité qu'ils prêtaient, et, si l'on prend en considération la lettre écrite par M. Aubusson au début du Directoire, qu'ils demeurèrent à leur poste lors des journées révolutionnaires, et qu'ils restèrent étrangers à tous les partis. On devra poursuivre avec plus de profondeur ces recherches, mais, pour l'instant, tout semble indiquer que les employés qui conservèrent leurs fonctions survécurent aux épurations ${ }^{91}$ et firent carrière sous la Révolution, s'engagèrent beaucoup plus dans leur travail et dans le parti pris pour l'institution dans laquelle ils travaillaient, et voulurent continuer à travailler, avec efficacité, intelligence et exactitude, que dans un des divers partis ou factions qu'il y eut pendant le processus révolutionnaire. 


\section{NOTES}

1. Voir par exemple les travaux de Raphaël MATTA-DUVIGnAU, Gouverner, administrer révolutionnairement: le comité de salut public (6 avril 1793 - 4 brumaire an IV), Paris, L'Harmattan, 2013 ; Julien Boudon, Les Jacobins. Une traduction des principes de Jean-Jacques Rousseau, préface de Frédéric Bluche, Thèse de Doctorat, Droit, Université Panthéon-Assas, 2002, Paris, L.G.D.J., 2006 ; Robert Roswell PALMER, Twelve who ruled, the committee of public safety during the Terror, Princeton, University Press, 1941 ; et Jacques CASTelnaU, Le comité de salut public, Paris, Librairie Hachette, 1941. Voir aussi, pour plus d'informations sur les travaux publiés concernant le Comité de salut public, Maria Betlem CASTELLÀ I PUJOLS, «Introduction. Que sait-on aujourd'hui des comités des assemblées parlementaires?», La Révolution française [En ligne], 3|2012, mis en ligne le 20 décembre 2012, Consulté le 11 octobre 2015. URL :

http://lrf.revues.org/674.

2. Ce parcours professionnel fut rédigé par M. Aubusson lui-même dans les premiers mois du Directoire. AN, AF III 20 A : Personnel des bureaux, an IV-an VII (microfilm).

3. Archives parlementaires des Chambres françaises de 1787 à 1860, recueil complet des débats législatifs et politiques des chambres françaises, première série (1787 à 1799) (maintenant AP), Paris, Paul Dupont éditeur, 1897, t. 52, p. 630.

4. Ibidem.

5. Ibidem.

6. Ibid., t. 34, p. 362.

7. À ce propos, lire Maurice GENTY, "Démocratie directe », dans Jean René Suratteau, François Gendron (dir.), Dictionnaire historique de la Révolution française, Paris, Presses Universitaires de France, 1989, p. 340-341.

8. Ibid., t. 52, p. 630-631.

9. Ibid., t. 52, p. 631.

10. Ibidem.

11. Ibidem.

12. Ibidem.

13. On peut lire dans le procès-verbal concernant la neuvième séance du Comité des pétitions et de correspondance ce qui suit : «Les citoyens Dechézeaux et Feraud ayant fait successivement les rapports dont ils avaient été chargés relativement au Bureau des renvois et de correspondance, le Comité arrête que ce bureau sera réuni à ceux existant déjà ou qui seront formés pour les diverses parties du travail qui lui est confié par le décret du onze de ce mois, conformément au plan d'organisation qui sera adopté et lors duquel ces commis qui y sont employés seront prévenus de la section à laquelle ils seront attachés à moins que les circonstances et le nouvel ordre de travail nécessitent la suppression de quelqu'un d'entre eux ", Archives Nationales, AF*II 18 : Comité des pétitions et de correspondance : procès-verbaux. 1792 (an II).

14. Voir l'annexe 1 de cet article.

15. Lire la neuvième séance du procès-verbal du Comité des pétitions et de correspondance. Archives Nationales, AF*II 18 : Comité des pétitions et de correspondance : procès-verbaux, 1792 (an II).

16. On peut lire dans le procès-verbal concernant la onzième séance du Comité des pétitions et de correspondance ce qui suit: «Le citoyen président a proposé d'organiser définitivement les sections du comité, et après de courtes observations faites par différents membres, l'organisation 
a été arrêtée ainsi qu'il suit. Art. $1^{\mathrm{er}}$ : Quatre des commis attachés au Bureau des renvois seront réunis à la section des pétitions pour faire la surveillance des membres de cette section, travailler à l'analyse des pétitions, adresses, lettres et mémoires adressés à la Convention et en faire l'envoi aux divers comités d'après les décrets de la Convention ou les arrêtés du Comité. Art. 2 : Les deux autres commis sous l'inspection des membres de la section de correspondance seront chargés de trier les lettres, pétitions et mémoires adressés par la poste à la Convention, de remettre au Président les objets dont il sera nécessaire de faire donner la lecture à l'Assemblée et de recueillir le reste pour être envoyé chaque jour à la section des pétitions, en conséquence le comité rapportant son arrêté du seize du courant en ce qui concerne la division du comité en trois sections arrête définitivement que ce jour le comité sera divisé seulement en deux sections, l'une des pétitions, l'autre de correspondance ", Archives Nationales, $\mathrm{AF}^{*} \mathrm{II} 18$ : Comité des pétitions et de correspondance : procès-verbaux, 1792 (an II).

17. Nous avons un doute en ce qui concerne M. Vaillant du Comité des pétitions et de correspondance, étant donné que dans le Comité des pétitions il y avait un secrétaire-commis appelé Vaillant et dans le Bureau des renvois de pièces il y avait aussi un secrétaire-commis nommé Vaillant (G. Vaillant). Avant de réaliser ce travail nous étions de l'avis que le Vaillant du Comité des pétitions et de correspondance était celui du Comité des pétitions, mais en tenant compte des dons patriotiques effectués et du fait que dans la section du Comité des pétitions il y avait seulement cinq secrétaires-commis en novembre 1792 et non six (Garnier, Hussenet, Chaulay, Dupuis et Chachoin), nous pensons que le Vaillant du Comité des pétitions et de correspondance était celui du Bureau des renvois de pièces. Voir l'annexe 1 de la première partie de cet article intitulée : Maria Betlem CASTELlÀ I PUJOLS, « Monsieur André Aubusson... », art. cit.

18. Voir l'annexe 1 de cet article.

19. Il y a quelques années Vida Azimi soulignait : « La recherche de l'efficacité et l'exigence de la technicité marquent encore la conduite de l'État révolutionnaire », Vida AZIMI, « Servir la nation : agents et fonctionnaires publics ", L'administration de la France sous la Révolution, Paris, Librairie Droz, 1992, p. 101.

20. Lire le procès-verbal de la douzième séance du Comité des pétitions et de correspondance. Archives Nationales, AF*II 18 : Comité des pétitions et de correspondance : procès-verbaux, 1792 (an II).

21. Ibidem.

22. Ibidem.

23. Ibidem.

24. Voir l'annexe 1 de cet article.

25. Ibidem.

26. Archives Nationales, $\mathrm{AF}^{*} \mathrm{II} 18$ : Comité des pétitions et de correspondance : procès-verbaux, 1792 (an II).

27. AP, t. 30, p. 17-18.

28. Voir l'annexe 1 de la première partie de cet article intitulée : « Monsieur André Aubusson... », art. cit.

29. AP, op. cit., t. 30, p. 24.

30. Maria Betlem CASTELLÀ I PUjols, Revolució, poder i informació. El control de la informació a les Assemblees parlamentàries durant la Revolució francesa (1789-1795), sous la direction de Lluís Roura i Aulinas et Jean-Clément Martin, Universitat Autònoma de Barcelona et Université Paris 1 Panthéon-Sorbonne, Bellaterra, 28 novembre 2008, t. 2, (mbcp2de4.pdf), p. 486-488.

31. Le 6 mars 1792, l'Assemblée législative adopta le décret suivant: «L'Assemblée nationale décrète qu'il sera formé immédiatement une commission chargée de recueillir et de lui présenter toutes les mesures propres au rétablissement et au maintien de la tranquillité publique. Cette commission sera composée de 2 membres de chacun des comités de pétition, d'agriculture, de commerce, de surveillance, militaire et de législation. L'Assemblée invite tous ses membres à 
communiquer à la commission tous les projets et toutes les vues qu'ils croiront utiles ", AP, op. cit. ,t. 39 , p. 428.

32. Le 17 juin 1792, les députés adoptèrent le décret qui suit: "L'Assemblée, consultée, décrète qu'il sera nommé, séance tenante, une commission de douze membres, pour examiner, sous tous les points de vue, l'état actuel de la France, en présenter le tableau sous huit jours et proposer les moyens de sauver la Constitution, la liberté et l'Empire. ", AP, op. cit., t. 45, p. 327.

33. Ibid., t. 61, p. 378.

34. François Alphonse AULARD, Recueil des actes du comité de salut public avec la correspondance officielle des représentants en mission et le registre du conseil exécutif provisoire, Paris, Imprimerie nationale, 1890, t. III, p. 133.

35. AP, op. cit., t. 61 , p. 449.

36. Ibid., t. 63, p. 558.

37. Ibidem.

38. Sur la tendance politique des membres du Comité des pétitions et du Comité de salut public, voir l'annexe 1 de l'article suivant: Maria Betlem CASTELlà i Pujols, "Métamorphoses d'un comité: le Comité des pétitions et de correspondance sous la Convention nationale ", La Révolution française [en ligne], 3 | 2012, mis en ligne le 20 décembre 2012, consulté le 13 juin 2014. URL : http://lrf.revues.org/702.

39. Voir l'annexe 2 de cet article.

40. Lire les pièces constituant le procès-verbal de la Commission des dépêches. Archives Nationales, $\mathrm{AF}^{*} \mathrm{II} 17$ : Commission des dépêches : procès-verbaux, 1793 (an III).

41. Selon le procès-verbal de la Commission des dépêches, le 9 avril 1793, les députés de cette commission arrêtèrent ce qui suit: "La Commission des Dépêches, nommée par décret de la Convention Nationale en date du 8 avril présent mois. Considérant que pour rendre compte de ses opérations il est nécessaire d'établir un registre d'ordre dans lequel toutes les Dépêches officielles seront inscrites; Considérant que les deux citoyens commis qui sont actuellement employés dans le bureau de correspondance sont insuffisants pour expédier le courant des affaires et tenir le dit Registre. Arrêtent que le citoyen Cheron, employé au Comité des Pétitions passera au Bureau de Correspondance pour tenir le dit Registre en conservant les appointements qui lui étoient précédemment alloués à raison du travail dont il étoit chargé au Comité des Pétitions. Délibéré par la commission des Dépêches de la Convention nationale le 9 avril 1793. L'an deux de la République française une et indivisible.", Archives Nationales, AF*II 17 : Commission des dépêches : procès-verbaux, 1793 (an III).

42. AP, op. cit., t. 30, p. 25.

43. Voir l'annexe 3 de cet article concernant les députés membres de la Commission des dépêches et leur présence réelle dans cette commission. Cette annexe a été réalisée à partir du procèsverbal de la Commission des dépêches. Archives Nationales, AF*II 17 : Commission des dépêches : procès-verbaux, 1793 (an III).

44. Arrêté pris à Paris le 28 juin 1793 (an II) de la République une et indivisible, avec les signatures de Rudel, Christiani, Périès et Guyardin, Archives Nationales, AF* II 17 : Commission des dépêches : procès-verbaux, 1793 (an III).

45. Décret pris à Paris le 17 août 1793 (an II) de la République une et indivisible, signé par Amar, Leonard Bourdon et Fayau, secrétaires de la Convention. Archives Nationales, AF*II 17 : Commission des dépêches : procès-verbaux, 1793 (an III).

46. AP, op. cit., t. 42, p. 282.

47. Ibid., t. 46, p. 120.

48. Ibid., t. 44, p. 491 (2 juin 1792) ; t. 46, p. 28 (2 juillet 1792); et t. 47, p. 393 (2 août 1792).

49. Ibid., t. 44, p. 6 (22 mai 1792).

50. Ibid., t. 44, p. 637 (6 juin 1792) ; t. 46, p. 556 (17 juillet 1792) ; et t. 47, p. 308 (31 juillet 1792).

51. Ibid., t. 48, p. 159. 
52. Selon les AP, le 15 août 1792 : «MM. M. Ducroisi, Braille, Prateau, Vanerelle, Behaigne, Renvoizé, Thibault, Henry, Atrux, Aubusson, Gautier, Leclerc, Villiers jeune, Viguier, Bourgoin, Jacquemel, Philidor, Cousin, Besson, Rosier, Lesguille, G. Vaillant, Leharivel, et Villiers aîné, secrétaires-commis attachés au Secrétariat de l'Assemblée, se présentent à la barre pour prêter le serment de maintenir la liberté et l'égalité, ou de mourir en les défendant. M. le Président applaudit leur civisme et leur accorde les honneurs de la séance », AP, op. cit., t. 48, p. 159.

53. Le 5 novembre 1792: "Les citoyens Vaillant, Renvoizé, Aubusson, Rimonneau, Atrux, Thibault et Henry donnent, pour le mois d'octobre, chacun un assignat de 5 livres. ", AP, op. cit., t. 53, p. 151.

54. Le 5 mars 1792, la lettre suivante fut lue à la Convention nationale: «Lettre du citoyen Aubusson, secrétaire-commis au Bureau de correspondance de la Convention nationale, qui s'est engagé à contribuer de 60 livres par an pour les frais de guerre, et dépose sur l'autel de la patrie trois assignats de cinq livres, pour les mois de décembre 1792, janvier et février 1793. ", AP, op. cit ., t. 59, p. 612.

55. On aimerait remercier ici les évaluateurs scientifiques de cet article pour leurs commentaires pertinents.

56. Le 13 septembre 1792, la Convention nationale adopta le décret suivant: "La Convention nationale, sur la proposition d'un membre, décrète que tous les comités, à l'exception de celui de salut public, seront renouvelés; charge son Comité de salut public de lui présenter une liste de candidats pour chacun d'eux. ", AP, op. cit., t. 74, p. 52.

57. François-Alphonse AULARD, Recueil des actes du comité de salut public avec la correspondance officielle des représentants en mission et le registre du conseil exécutif provisoire, op. cit., t. VI, p. 459.

58. Raphaël MATTA-DUVIGNAU, Gouverner, administrer révolutionnairement: le comité de salut public (6 avril 1793 - 4 brumaire an IV), op. cit., p. 222.

59. À propos des salaires des employés, voir Vida AZIMI, « Heur et malheur des "salariés publics" sous la Révolution ", dans État, finances et économie pendant la Révolution française, Paris, Comité pour l'histoire économique et financière de la France, 1991, p. 159-200.

60. Ibid., p. 314-315.

61. François WARTELLE, «Le Bon Joseph", dans Jean René Suratteau, François Gendron (dir.), Dictionnaire historique de la Révolution française, op. cit., p. 656.

62. Avant la nomination de Maximilien Robespierre au Comité de salut public, on avait nommé les députés suivants : Cambon, Guyton-Morveau, Danton, Barère, Delmas, Delacroix, Treilhard, Bréard, Lindet, Couthon, Hérault de Séchelles, Mathieu, Ramel-Nogaret, Saint-Just, Berlier, Gasparin, Jeanbon Saint-André, Mallarmé, Lindet, Du Roy, Francastel, Prieur de la Marne et Thuriot.

63. Le 27 juillet 1793, Maximilien Robespierre remplaça Gasparin au Comité de salut public. AP, op. cit., t. 69 , p. 584 .

64. Maximilien ROBESPIERRE, Euvres de Maximilien Robespierre, Paris, Phénix Éditions, 2000, t. IX, p. 560.

65. Ibid., p. 614

66. Ibid., t. X, p. 46.

67. Ibid., t. X, p. 85.

68. Ibid., t. X, p. 80-81.

69. Raphaël MATTA-DUVIGNAU, Gouverner, administrer révolutionnairement: le comité de salut public (6 avril 1793 - 4 brumaire an IV), op. cit., p. 594.

70. AN, AF III 20 A, Personnel des bureaux, an IV-an VII (microfilm).

71. François Alphonse AULARD, Recueil des actes du comité de salut public avec la correspondance officielle des représentants en mission et le registre du conseil exécutif provisoire, op. cit., t. VI, p. 575.

72. AN, AF III 20 A, Personnel des bureaux, an IV-an VII (microfilm). 
73. D'abord dans le Bureau de correspondance de l'Assemblée nationale constituante, ensuite dans le Bureau des renvois et de correspondance de l'Assemblée législative et, finalement, dans la Section des renvois et dons patriotiques du Comité des pétitions et de correspondances et dans la Commission des dépêches.

74. François Alphonse AULARD, Recueil des actes du comité de salut public avec la correspondance officielle des représentants en mission et le registre du conseil exécutif provisoire, op. cit., t. XIII, p. 110 ; t. XIX, p. 376, p. 427 ; t. XXIV, p. 496-497 ; t. XXV, p. 160.

75. AN, AF III 20 A, Personnel des bureaux, an IV-an VII (microfilm).

76. Ibidem.

77. Ibidem.

78. Ibidem.

79. Ibidem.

80. AN, AF III 537, plaquette 3549, partie du 24 thermidor. À la date du 12 fructidor an VI, Monsieur Lagarde reprit son poste. Pierre Dominique CHEYNET, Procès-verbaux $d u$ Directoire exécutif, an V-an VIII : inventaire des registres des délibérations et des minutes des arrêtés, lettres et actes $d u$ Directoire, Paris, Centre historique des Archives nationales, 2003, t. VI, p. 31. Consulté en ligne le 14 juin 2014.

81. Ibid., t. IX, p. 42. À la date du 16 prairial an VII, M. Aubusson était encore secrétaire général du Directoire. Ibid., t. IX, p. 56.

82. AN, AF III 20 A, Personnel des bureaux, an IV-an VII (microfilm).

83. Le 7 frimaire an IV (28 novembre 1795) le Directoire exécutif arrêta ce qui suit : «Art. $1^{\mathrm{er}}$ : À compter du $1^{\text {er }}$ frimaire, les traitements des employés des administrations publiques sont fixés en valeur de 1790, et payés en assignats à raison de trente fois la somme. Art. 2 : Les traitements des employés du Directoire, des ministères ou administrations publiques, résidant à Paris, y compris les préposés de ces administrations, qui sont dans les départements, seront établis de la manière suivante: $1^{\text {o }}$ Chefs directeurs de grandes divisions, membres des agences et administrations secondaires, huit mille livres à six mille livres [...]». Dix jours plus tard, le 17 frimaire an IV (8 décembre 1795), le Directoire exécutif arrêta le suivant: «Art. $1^{\mathrm{er}}$ : Les employés mentionnés en l'article 2 de l'arrêté du 7 frimaire seront divisés en quatre classes. Première classe. Le traitement des membres des agences, secrétaires en chef, chefs ou directeurs de divisions et autres à ranger dans la première classe, ne pourra être élevé provisoirement au-dessus de douze mille livres par mois, ce qui fait six mille livres par quinzaine [...]», Jean Baptiste DUVERGIER, Collection complète des lois, décrets, ordonnances, règlements et avis du Conseil d'État, Paris, chez A. Guyot et Scribe, 1825, t. IX, p. 13 et 18.

84. AN, AF III 20 A, Personnel des bureaux, an IV-an VII (microfilm).

85. AN, AF III 24, Serments des employés, an IV-an VII (microfilm).

86. Pierre Dominique CHEYNET, Procès-verbaux du Directoire exécutif, an V-an VIII : inventaire des registres des délibérations et des minutes des arrêtés, lettres et actes du Directoire, op. cit., t. IX, p. 9.

87. C'est le cas des citoyens Renvoizé, Doumengeat, Fourcade, Garnier, Chachoin et Atrux, secrétaires commis du Comité des pétitions et de correspondance. Voir l'annexe 1 de la première partie de cet article déjà publiée : « Monsieur André Aubusson...», op. cit.

88. Raphaël MATTA-DUVIGNAU, Gouverner, administrer révolutionnairement... op. cit., p. 350.

89. Jean-René SURATTEAU, «Directoire (Le)», dans Jean René Suratteau, François Gendron (dir.), Dictionnaire historique de la Révolution française, op. cit., p. 355.

90. Catherine KAWA, Les Ronds-de-cuir en Révolution. Les employés du ministère de l'Intérieur sous la Première République (1792-1800), Paris, Éditions du CTHS, 1996, p. 261.

91. Voir Clive H. CHURCH, «Bureacracy, Politics, and Revolution: The évidence of the Commission des Dix-sept », French Historial Studies, vol. 6, n4, p. 492-516. 


\section{RÉSUMÉS}

En suivant l'ascension professionnelle d'un ancien militaire, M. André Aubusson, qui commença à gérer la correspondance reçue par le corps législatif dès les premiers jours du processus révolutionnaire, fût nommé secrétaire principal du Comité de salut public au mois de septembre 1793 et finit par devenir le chef de la division du Secrétariat général du Directoire exécutif le 13 brumaire de l'an IV, nous voulons expliquer dans cet article, qui est en fait la deuxième partie d'un autre travail publié sous le titre : « Monsieur André Aubusson, l'homme qui sait tout, ou gérer la correspondance sous les assemblées parlementaires », qui gérait la correspondance, et comment, sous la première année de la République, l'impact que l'établissement du Comité de salut public eut sur les filtres établis sur la correspondance, le parcours professionnel de M. Aubusson sous la Convention nationale et le Directoire ainsi que la continuité qu'on peut apprécier d'un même personnel administratif dans le cadre des bureaux et des comités qui s'occupent de gérer la correspondance sous les différentes assemblées parlementaires. Les députés sont renouvelés de temps en temps, mais les secrétaires-commis qui y travaillent, certains d'entre eux depuis les premiers jours de l'Assemblée nationale constituante, ne le sont pas, configurant ainsi un corps d'employés expérimentés qui conservent leurs fonctions au long des trois premières assemblées, et parfois, dans les années qui suivent.

In following the professional progress of a former military man, André Aubusson, who began to take charge of the correspondence received by the legislature in the first days of the revolutionary process, who was appointed the principal secretary of the Committee of Public Safety in September 1793, and who went on to become section head of the General Secretariat of the executive Directory on 13 Brumaire of Year IV, the purpose of the present article-which is in fact the second part of an earlier study published under the title "Monsieur André Aubusson, the man who knew everything, or the control of the correspondence under the parliamentary assemblies", is to explain who managed the correspondence, and how, during the first year of the Republic; the impact that the setting up of the Committee of Public Safety had on the filters put in place to manage that correspondence; the professional career of Mr. Aubusson in the National Convention and the Executive Directory, and the continuity that can be identified within the body of administrative staff in the offices and committees responsible for managing correspondence under the different parliamentary assemblies. The deputies were replaced from time to time, but the secretary-clerks who worked there, as some of them had since the first days of the Constituent Assembly, were not, and thus constituted a corps of experienced personnel who continued to perform their functions throughout the first three Assemblies, and in some cases, in the years that followed. 
INDEX

Keywords : André Aubusson, Secretary-clerks, Committee on Petitions and Correspondence, Committee of Public Safety, Commission of Dispatches, General Secretariat of the executive Directory

Mots-clés : André Aubusson, secrétaires, commis, Comité des pétitions et de correspondance, Comité de salut public, Commission des dépêches, Secrétariat général du Directoire exécutif

\section{AUTEUR}

\section{MARIA BETLEM CASTELLÀ I PUJOLS}

Universitat Pompeu Fabra 